\title{
Unitarity Quadrangles of Four Neutrino Mixing
}

\author{
Wan-lei Guo and Zhi-zhong Xing \\ CCAST (World Laboratory), P.O. Box 8730, Beijing 100080, China; \\ and Institute of High Energy Physics, Chinese Academy of Sciences, \\ P.O. Box 918 (4), Beijing 100039, China \\ (Electronic address: guowl@mail.ihep.ac.cn; xingzz@mail.ihep.ac.cn)
}

\begin{abstract}
We present a classification of the unitarity quadrangles in the four-neutrino mixing scheme. We find that there are totally thirty-six distinct topologies among twelve different unitarity quadrangles. Concise relations are established between the areas of those unitarity quadrangles and the rephasing invariants of $C P$ and $T$ violation.
\end{abstract}

PACS number(s): 14.60.Pq, 13.10.+q, 25.30.Pt

*Mailing address 
The robust Super-Kamiokande [1] and SNO [2] data have provided convincing evidence that the atmospheric $\nu_{\mu}$ neutrinos convert primarily into $\nu_{\tau}$ neutrinos, while the solar $\nu_{e}$ neutrinos convert essentially into $\nu_{\mu}$ or $\nu_{\tau}$ neutrinos. It turns out that the existence of a light sterile neutrino $\nu_{s}$, which has been assumed to reconcile the LSND [3] evidence for $\bar{\nu}_{\mu} \rightarrow \bar{\nu}_{e}$ (and $\nu_{\mu} \rightarrow \nu_{e}$ ) oscillations with solar and atmospheric neutrino data in the fourneutrino mixing scenarios 四 1 , becomes questionable. Indeed, a recent globable analysis of current neutrino oscillation data has shown that the well-known $(2+2)$ and $(3+1)$ schemes of four neutrino mixing are both disfavored [7]. The upcoming MiniBooNE experiment [8] is therefore crucial, in order to confirm or disprove the LSND measurement. Before a definitely negative conclusion can be drawn from MiniBooNE, however, the LSND data should be taken seriously. In particular, it is worthwhile to investigate the four-neutrino mixing scenarios in a way without special theoretical biases and (or) empirical assumptions.

In a recent paper [9], we have calculated the rephasing invariants of $C P$ and $T$ violation by use of a favorable or "standard" parametrization of the generic $4 \times 4$ neutrino mixing matrix. Our results are expected to be quite useful for a systematical analysis of $C P$ - and $T$-violating effects in various long-baseline neutrino oscillation experiments. In the present work, which may serve as an important addendum to Ref. [9], we aim to present a complete geometrical description of $C P$ and $T$ violation in the four-neutrino mixing scheme.

It is well known that the language of unitarity triangles is very helpful for the description of $C P$ violation in the quark sector [10]. The same language has been introduced into the lepton sector to describe $C P$ violation in the framework of three-family lepton flavor mixing [11,12, 13, 14]. In Ref. [12], an example concerning the unitarity quadrangles is given to illustrate the necessary condition of $C P$ violation in the four-neutrino mixing scenario 2 . In this Brief Report, we shall first make a classification of all possible unitarity quadrangles and then calculate their areas in terms of the rephasing invariants of $C P$ violation based on the standard parametrization of four neutrino mixing.

Let us consider the admixture of three active $\left(\nu_{e}, \nu_{\mu}, \nu_{\tau}\right)$ neutrinos and one sterile $\left(\nu_{s}\right)$ neutrino. Although $\nu_{s}$ does not participate in any normal weak interactions, it may oscillate with $\nu_{e}, \nu_{\mu}$ and $\nu_{\tau}$ [15]. Hence a $4 \times 4$ unitary matrix $V$ is required to fully describe four neutrino mixing in neutrino oscillations. In the basis where the flavor and mass eigenstates of charged leptons are identical, $V$ is defined to link the neutrino mass eigenstates $\left(\nu_{0}, \nu_{1}, \nu_{2}, \nu_{3}\right)$ to the neutrino flavor eigenstates $\left(\nu_{s}, \nu_{e}, \nu_{\mu}, \nu_{\tau}\right)$ :

$$
\left(\begin{array}{c}
\nu_{s} \\
\nu_{e} \\
\nu_{\mu} \\
\nu_{\tau}
\end{array}\right)=\left(\begin{array}{cccc}
V_{s 0} & V_{s 1} & V_{s 2} & V_{s 3} \\
V_{e 0} & V_{e 1} & V_{e 2} & V_{e 3} \\
V_{\mu 0} & V_{\mu 1} & V_{\mu 2} & V_{\mu 3} \\
V_{\tau 0} & V_{\tau 1} & V_{\tau 2} & V_{\tau 3}
\end{array}\right)\left(\begin{array}{c}
\nu_{0} \\
\nu_{1} \\
\nu_{2} \\
\nu_{3}
\end{array}\right) .
$$

\footnotetext{
${ }^{1}$ Instead of introducing a light sterile neutrino, a few more far-fetched ideas (such as the violation of CPT symmetry in the neutrino sector [5] and the lepton-number-violating muon decay [6]) have been proposed in the literature.

${ }^{2}$ The unitarity quadrangle discussed in Ref. [12] is equivalent to unitarity quadrangle $\mathrm{Q}_{e \mu}$ defined by us in Eq. (3).
} 
As neutrinos are expected to be Majorana particles, a full parametrization of $V$ needs six mixing angles and six $C P$-violating phases. Here we make use of the standard parametrization advocated in Ref. [16]; i.e.,

$$
V=\left(\begin{array}{cccc}
c_{01} c_{02} c_{03} & c_{02} c_{03} \hat{s}_{01}^{*} & c_{03} \hat{s}_{02}^{*} & \hat{s}_{03}^{*} \\
-c_{01} c_{02} \hat{s}_{03} \hat{s}_{13}^{*} & -c_{02} \hat{s}_{01}^{*} \hat{s}_{03} \hat{s}_{13}^{*} & -\hat{s}_{02}^{*} \hat{s}_{03} \hat{s}_{13}^{*} & c_{03} \hat{s}_{13}^{*} \\
-c_{01} c_{13} \hat{s}_{02} \hat{s}_{12}^{*} & -c_{13} \hat{s}_{01}^{*} \hat{s}_{02} \hat{s}_{12}^{*} & +c_{02} c_{13} \hat{s}_{12}^{*} & \\
-c_{12} c_{13} \hat{s}_{01} & +c_{01} c_{12} c_{13} & & \\
& & & \\
-c_{01} c_{02} c_{13} \hat{s}_{03} \hat{s}_{23}^{*} & -c_{02} c_{13} \hat{s}_{01}^{*} \hat{s}_{03} \hat{s}_{23}^{*} & -c_{13} \hat{s}_{02}^{*} \hat{s}_{03} \hat{s}_{23}^{*} & c_{03} c_{13} \hat{s}_{23}^{*} \\
+c_{01} \hat{s}_{02} \hat{s}_{12}^{*} \hat{s}_{13} \hat{s}_{23}^{*} & +\hat{s}_{01}^{*} \hat{s}_{02} \hat{s}_{12}^{*} \hat{s}_{13} \hat{s}_{23}^{*} & -c_{02} \hat{s}_{12}^{*} \hat{s}_{13} \hat{s}_{23}^{*} & \\
-c_{01} c_{12} c_{23} \hat{s}_{02} & -c_{12} c_{23} \hat{s}_{01}^{*} \hat{s}_{02} & +c_{02} c_{12} c_{23} & \\
+c_{12} \hat{s}_{01} \hat{s}_{13} \hat{s}_{23}^{*} & -c_{01} c_{12} \hat{s}_{13} \hat{s}_{23}^{*} & & \\
+c_{23} \hat{s}_{01} \hat{s}_{12} & -c_{01} c_{23} \hat{s}_{12} & & \\
-c_{01} c_{02} c_{13} c_{23} \hat{s}_{03} & -c_{02} c_{13} c_{23} \hat{s}_{01}^{*} \hat{s}_{03} & -c_{13} c_{23} \hat{s}_{02}^{*} \hat{s}_{03} & c_{03} c_{13} c_{23} \\
+c_{01} c_{23} \hat{s}_{02} \hat{s}_{12}^{*} \hat{s}_{13} & +c_{23} \hat{s}_{01}^{*} \hat{s}_{02} \hat{s}_{12}^{*} \hat{s}_{13} & -c_{02} c_{23} \hat{s}_{12}^{*} \hat{s}_{13} & \\
+c_{01} c_{12} \hat{s}_{02} \hat{s}_{23} & +c_{12} \hat{s}_{01}^{*} \hat{s}_{02} \hat{s}_{23} & -c_{02} c_{12} \hat{s}_{23} & \\
+c_{12} c_{23} \hat{s}_{01} \hat{s}_{13} & -c_{01} c_{12} c_{23} \hat{s}_{13} & & \\
-\hat{s}_{01} \hat{s}_{12} \hat{s}_{23} & +c_{01} \hat{s}_{12} \hat{s}_{23} & &
\end{array}\right)
$$

where $c_{i j} \equiv \cos \theta_{i j}$ and $\hat{s}_{i j} \equiv s_{i j} e^{\mathrm{i} \delta_{i j}}$ with $s_{i j} \equiv \sin \theta_{i j}$. The strength of $C P$ and $T$ violation in neutrino oscillations is governed by the Jarlskog invariants $J_{\alpha \beta}^{i j} \equiv \operatorname{Im}\left(V_{\alpha i} V_{\beta j} V_{\alpha j}^{*} V_{\beta i}^{*}\right)$ [17], where the Greek subscripts run over $(s, e, \mu, \tau)$ and the Latin superscripts run over $(0,1,2,3)$.

Note that a generic $n \times n$ lepton flavor mixing matrix consists of $n(n-1) / 2$ mixing angles, $(n-1)(n-2) / 2$ Dirac-type phases and $(n-1)$ Majorana-type phases [11, 18]. Furthermore, the number of independent Jarlskog invariants is $(n-1)^{2}(n-2)^{2} / 4$ [19]. For $n=4$, we arrive at nine independent Jarlskog invariants compared to three Dirac-type $C P$-violating phases in $V$. This implies that there is no one-by-one correspondence between Jarlskog parameters and Dirac-type phases, if the number of lepton families is equal to or larger than four. Given the parametrization of $V$ in Eq. (2) for four neutrino mixing, nine independent $J_{\alpha \beta}^{i j}$ are actually functions of three phase combinations (of the Dirac nature) and six mixing angles, as explicitly shown in Ref. [9].

The unitarity of $V$ implies that there are twelve orthogonality relations and eight normalization conditions among its sixteen matrix elements. The former corresponds to twelve quadrangles in the complex plane, the so-called unitarity quadrangles. To be explicit, let us write out the twelve orthogonality relations and name their corresponding quadrangles:

$$
\begin{array}{ll}
\mathrm{Q}_{s e}: & V_{s 0} V_{e 0}^{*}+V_{s 1} V_{e 1}^{*}+V_{s 2} V_{e 2}^{*}+V_{s 3} V_{e 3}^{*}=0, \\
\mathrm{Q}_{s \mu}: & V_{s 0} V_{\mu 0}^{*}+V_{s 1} V_{\mu 1}^{*}+V_{s 2} V_{\mu 2}^{*}+V_{s 3} V_{\mu 3}^{*}=0, \\
\mathrm{Q}_{s \tau}: & V_{s 0} V_{\tau 0}^{*}+V_{s 1} V_{\tau 1}^{*}+V_{s 2} V_{\tau 2}^{*}+V_{s 3} V_{\tau 3}^{*}=0, \\
\mathrm{Q}_{e \mu}: & V_{e 0} V_{\mu 0}^{*}+V_{e 1} V_{\mu 1}^{*}+V_{e 2} V_{\mu 2}^{*}+V_{e 3} V_{\mu 3}^{*}=0, \\
\mathrm{Q}_{e \tau}: & V_{e 0} V_{\tau 0}^{*}+V_{e 1} V_{\tau 1}^{*}+V_{e 2} V_{\tau 2}^{*}+V_{e 3} V_{\tau 3}^{*}=0, \\
\mathrm{Q}_{\mu \tau}: & V_{\mu 0} V_{\tau 0}^{*}+V_{\mu 1} V_{\tau 1}^{*}+V_{\mu 2} V_{\tau 2}^{*}+V_{\mu 3} V_{\tau 3}^{*}=0 ;
\end{array}
$$


and

$$
\begin{aligned}
& \mathrm{Q}_{01}: V_{s 0} V_{s 1}^{*}+V_{e 0} V_{e 1}^{*}+V_{\mu 0} V_{\mu 1}^{*}+V_{\tau 0} V_{\tau 1}^{*}=0, \\
& \mathrm{Q}_{02}: V_{s 0} V_{s 2}^{*}+V_{e 0} V_{e 2}^{*}+V_{\mu 0} V_{\mu 2}^{*}+V_{\tau 0} V_{\tau 2}^{*}=0, \\
& \mathrm{Q}_{03}: V_{s 0} V_{s 3}^{*}+V_{e 0} V_{e 3}^{*}+V_{\mu 0} V_{\mu 3}^{*}+V_{\tau 0} V_{\tau 3}^{*}=0, \\
& \mathrm{Q}_{12}: V_{s 1} V_{s 2}^{*}+V_{e 1} V_{e 2}^{*}+V_{\mu 1} V_{\mu 2}^{*}+V_{\tau 1} V_{\tau 2}^{*}=0, \\
& \mathrm{Q}_{13}: V_{s 1} V_{s 3}^{*}+V_{e 1} V_{e 3}^{*}+V_{\mu 1} V_{\mu 3}^{*}+V_{\tau 1} V_{\tau 3}^{*}=0, \\
& \mathrm{Q}_{23}: V_{s 2} V_{s 3}^{*}+V_{e 2} V_{e 3}^{*}+V_{\mu 2} V_{\mu 3}^{*}+V_{\tau 2} V_{\tau 3}^{*}=0 .
\end{aligned}
$$

If six mixing angles and six $C P$-violating phases of $V$ are all known, one can plot twelve unitarity quadrangles without ambiguities. Note, however, that each quadrangle has three distinct topologies in the complex plane. For illustration, we take quadrangle $\mathrm{Q}_{\text {se }}$ for example and show its three topologies in FIG. 1, where the sizes and phases of $V_{s i} V_{e i}^{*}$ (for $i=0,1,2,3$ ) have been fixed. One can see that different topologies of quadrangle $\mathrm{Q}_{s e}$ arise from different orderings of its four sides, and their areas are apparently different from one another. As a whole, there are totally thirty-six different topologies among twelve unitarity quadrangles.

Now we calculate the areas of all unitarity triangles and relate them to the rephasing invariants of $C P$ violation $J_{\alpha \beta}^{i j}$. Taking quadrangle $\mathrm{Q}_{s e}$ as an example again, we find that the areas of its three distinct topologies can be given respectively by $\mathrm{B}$

$$
\begin{aligned}
S_{s e}^{a} & =\frac{1}{4}\left[\operatorname{Im}\left(V_{s 1} V_{e 0} V_{s 0}^{*} V_{e 1}^{*}\right)+\operatorname{Im}\left(V_{s 2} V_{e 1} V_{s 1}^{*} V_{e 2}^{*}\right)+\operatorname{Im}\left(V_{s 3} V_{e 2} V_{s 2}^{*} V_{e 3}^{*}\right)+\operatorname{Im}\left(V_{s 0} V_{e 3} V_{s 3}^{*} V_{e 0}^{*}\right)\right] \\
& =\frac{1}{4}\left(J_{s e}^{10}+J_{s e}^{21}+J_{s e}^{32}+J_{s e}^{03}\right), \\
S_{s e}^{b} & =\frac{1}{4}\left[\operatorname{Im}\left(V_{s 2} V_{e 0} V_{s 0}^{*} V_{e 2}^{*}\right)+\operatorname{Im}\left(V_{s 1} V_{e 2} V_{s 2}^{*} V_{e 1}^{*}\right)+\operatorname{Im}\left(V_{s 3} V_{e 1} V_{s 1}^{*} V_{e 3}^{*}\right)+\operatorname{Im}\left(V_{s 0} V_{e 3} V_{s 3}^{*} V_{e 0}^{*}\right)\right] \\
& =\frac{1}{4}\left(J_{s e}^{20}+J_{s e}^{12}+J_{s e}^{31}+J_{s e}^{03}\right), \\
S_{s e}^{c} & =\frac{1}{4}\left[\operatorname{Im}\left(V_{s 1} V_{e 0} V_{s 0}^{*} V_{e 1}^{*}\right)+\operatorname{Im}\left(V_{s 3} V_{e 1} V_{s 1}^{*} V_{e 3}^{*}\right)+\operatorname{Im}\left(V_{s 2} V_{e 3} V_{s 3}^{*} V_{e 2}^{*}\right)+\operatorname{Im}\left(V_{s 0} V_{e 2} V_{s 2}^{*} V_{e 0}^{*}\right)\right] \\
& =\frac{1}{4}\left(J_{s e}^{10}+J_{s e}^{31}+J_{s e}^{23}+J_{s e}^{02}\right),
\end{aligned}
$$

where $J_{s e}^{i j}$ have been defined below Eq. (2). In a similar way, one may calculate the areas of the other eleven unitarity quadrangles. The results for thirty-six different topologies of twelve unitarity quadrangles are summarized as follows:

\footnotetext{
${ }^{3}$ It should be noted that the areas of unitarity quadrangles under discussion are "algebraic areas", namely, they can be either positive or negative. Of course, it is always possible to take $S_{s e}^{a}=$ $\left(\left|J_{s e}^{10}\right|+\left|J_{s e}^{21}\right|+\left|J_{s e}^{32}\right|+\left|J_{s e}^{03}\right|\right) / 4$ or $S_{s e}^{a}=\left|J_{s e}^{10}+J_{s e}^{21}+J_{s e}^{32}+J_{s e}^{03}\right| / 4$, such that $S_{s e}^{a}$ is definitely positive. We find, however, that the language of "algebraic areas" is simpler and more convenient in the description of unitarty quadrangles. In particular, the algebraic area of unitarity quadrangle $\mathrm{Q}_{s e}$ in the case of topology (b) means an algebraic sum of the areas of its two disassociated triangles, which have opposite signs. Hence both $S_{s e}^{b}=0$ and $S_{s e}^{b}<0$ are in general allowed. As for topologies (a) and (c) of $\mathrm{Q}_{s e}, S_{s e}^{a}>0$ and $S_{s e}^{c}>0$ are simply a matter of sign or phase convention.
} 


$$
\begin{aligned}
S_{\alpha \beta}^{a} & =\frac{1}{4}\left(J_{\alpha \beta}^{10}+J_{\alpha \beta}^{21}+J_{\alpha \beta}^{32}+J_{\alpha \beta}^{03}\right), \\
S_{i j}^{a} & =\frac{1}{4}\left(J_{e s}^{i j}+J_{\mu e}^{i j}+J_{\tau \mu}^{i j}+J_{s \tau}^{i j}\right) ; \\
S_{\alpha \beta}^{b} & =\frac{1}{4}\left(J_{\alpha \beta}^{20}+J_{\alpha \beta}^{12}+J_{\alpha \beta}^{31}+J_{\alpha \beta}^{03}\right), \\
S_{i j}^{b} & =\frac{1}{4}\left(J_{\mu s}^{i j}+J_{e \mu}^{i j}+J_{\tau e}^{i j}+J_{s \tau}^{i j}\right) ;
\end{aligned}
$$

and

$$
\begin{aligned}
S_{\alpha \beta}^{c} & =\frac{1}{4}\left(J_{\alpha \beta}^{10}+J_{\alpha \beta}^{31}+J_{\alpha \beta}^{23}+J_{\alpha \beta}^{02}\right), \\
S_{i j}^{c} & =\frac{1}{4}\left(J_{e s}^{i j}+J_{\tau e}^{i j}+J_{\mu \tau}^{i j}+J_{s \mu}^{i j}\right),
\end{aligned}
$$

where the subscripts $\alpha \beta=s e, s \mu, s \tau, e \mu$, e $\tau$ or $\mu \tau$, and $i j=01,02,03,12,13$ or 23. Note that the correlation of $J_{\alpha \beta}^{i j}$ [9,16] allows us to simplify Eqs. (6), (7) and (8). Then each $S_{\alpha \beta}^{q}$ or $S_{i j}^{q}$ (for $\left.q=a, b, c\right)$ can be expressed as a sum of two independent Jarlskog invariants. Such simplified expressions of $S_{\alpha \beta}^{q}$ and $S_{i j}^{q}$ depend on the choice of independent $J_{\alpha \beta}^{i j}$, therefore they may have many different forms. If nine independent Jarlskog invariants are fixed, however, some expressions of $S_{\alpha \beta}^{q}$ and $S_{i j}^{q}$ must consist of three $J_{\alpha \beta}^{i j}$. This point will become clear later on.

Inversely, one may express $J_{\alpha \beta}^{i j}$ in terms of $S_{\alpha \beta}^{q}$ or $S_{i j}^{q}$. The explicit formulas are

$$
\left(\begin{array}{c}
J_{\alpha \beta}^{01} \\
J_{\alpha \beta}^{02} \\
J_{\alpha \beta}^{03} \\
J_{\alpha \beta}^{12} \\
J_{\alpha \beta}^{13} \\
J_{\alpha \beta}^{23}
\end{array}\right)=\left(\begin{array}{ccc}
-1 & 0 & -1 \\
0 & -1 & 1 \\
1 & 1 & 0 \\
-1 & 1 & 0 \\
0 & -1 & -1 \\
-1 & 0 & 1
\end{array}\right)\left(\begin{array}{c}
S_{\alpha \beta}^{a} \\
\\
S_{\alpha \beta}^{b} \\
S_{\alpha \beta}^{c}
\end{array}\right)
$$

which corresponds to the unitarity quadrangles $\mathrm{Q}_{s e}, \mathrm{Q}_{s \mu}, \mathrm{Q}_{s \tau}, \mathrm{Q}_{e \mu}, \mathrm{Q}_{e \tau}$ and $\mathrm{Q}_{\mu \tau}$ defined in Eq. (3); and

$$
\left(\begin{array}{l}
J_{s e}^{i j} \\
J_{s \mu}^{i j} \\
J_{s \tau}^{i j} \\
J_{e \mu}^{i j} \\
J_{e \tau}^{i j} \\
J_{\mu \tau}^{i j}
\end{array}\right)=\left(\begin{array}{ccc}
-1 & 0 & -1 \\
0 & -1 & 1 \\
1 & 1 & 0 \\
-1 & 1 & 0 \\
0 & -1 & -1 \\
-1 & 0 & 1
\end{array}\right)\left(\begin{array}{c}
S_{i j}^{a} \\
\\
S_{i j}^{b} \\
S_{i j}^{c}
\end{array}\right)
$$

which corresponds to the unitarity quadrangles $\mathrm{Q}_{01}, \mathrm{Q}_{02}, \mathrm{Q}_{03}, \mathrm{Q}_{12}, \mathrm{Q}_{13}$ and $\mathrm{Q}_{23}$ defined in Eq. (4).

As $J_{\alpha \beta}^{i j}=-J_{\alpha \beta}^{j i}=-J_{\beta \alpha}^{i j}=J_{\beta \alpha}^{j i}$ holds by definition, one may easily obtain $S_{\alpha \beta}^{q}=-S_{\beta \alpha}^{q}$ and $S_{i j}^{q}=-S_{j i}^{q}($ for $q=a, b, c)$. From the sum rule [9, 16] 


$$
\sum_{i} J_{\alpha \beta}^{i j}=\sum_{j} J_{\alpha \beta}^{i j}=\sum_{\alpha} J_{\alpha \beta}^{i j}=\sum_{\beta} J_{\alpha \beta}^{i j}=0
$$

one can also find

$$
\sum_{\alpha} S_{\alpha \beta}^{q}=\sum_{\beta} S_{\alpha \beta}^{q}=\sum_{i} S_{i j}^{q}=\sum_{j} S_{i j}^{q}=0
$$

where $\alpha$ or $\beta$ runs over $(s, e, \mu, \tau)$, and $i$ or $j$ runs over $(0,1,2,3)$. In addition to Eq. (12), the following relations can be derived from Eqs. (6), (7) and (8):

$$
\begin{aligned}
& -S_{s e}^{a}-S_{e \mu}^{a}-S_{\mu \tau}^{a}+S_{s \tau}^{a}=-S_{01}^{a}-S_{12}^{a}-S_{23}^{a}+S_{03}^{a}, \\
& -S_{s \mu}^{a}+S_{e \mu}^{a}-S_{e \tau}^{a}+S_{s \tau}^{a}=-S_{01}^{b}-S_{12}^{b}-S_{23}^{b}+S_{03}^{b}, \\
& -S_{s e}^{a}-S_{e \tau}^{a}+S_{\mu \tau}^{a}+S_{s \mu}^{a}=-S_{01}^{c}-S_{12}^{c}-S_{23}^{c}+S_{03}^{c} ; \\
& -S_{s e}^{b}-S_{e \mu}^{b}-S_{\mu \tau}^{b}+S_{s \tau}^{b}=-S_{02}^{a}+S_{12}^{a}-S_{13}^{a}+S_{03}^{a}, \\
& -S_{s \mu}^{b}+S_{e \mu}^{b}-S_{e \tau}^{b}+S_{s \tau}^{b}=-S_{02}^{b}+S_{12}^{b}-S_{13}^{b}+S_{03}^{b}, \\
& -S_{s e}^{b}-S_{e \tau}^{b}+S_{\mu \tau}^{b}+S_{s \mu}^{b}=-S_{02}^{c}+S_{12}^{c}-S_{13}^{c}+S_{03}^{c}
\end{aligned}
$$

and

$$
\begin{aligned}
& -S_{s e}^{c}-S_{e \mu}^{c}-S_{\mu \tau}^{c}+S_{s \tau}^{c}=-S_{01}^{a}-S_{13}^{a}+S_{23}^{a}+S_{02}^{a} \\
& -S_{s \mu}^{c}+S_{e \mu}^{c}-S_{e \tau}^{c}+S_{s \tau}^{c}=-S_{01}^{b}-S_{13}^{b}+S_{23}^{b}+S_{02}^{b} \\
& -S_{s e}^{c}-S_{e \tau}^{c}+S_{\mu \tau}^{c}+S_{s \mu}^{c}=-S_{01}^{c}-S_{13}^{c}+S_{23}^{c}+S_{02}^{c} .
\end{aligned}
$$

The correlation equations $(12)$ - (15) indicate that there are only nine independent $S_{\alpha \beta}^{q}$ and (or) $S_{i j}^{q}$, corresponding to nine independent $J_{\alpha \beta}^{i j}$.

Without loss of generality, let us choose the following nine independent $S_{\alpha \beta}^{q}$ :

$$
\begin{aligned}
& S_{s e}^{a}=\frac{1}{2}\left(J_{s e}^{02}-J_{s e}^{13}-2 J_{s e}^{23}\right), \\
& S_{s \tau}^{a}=\frac{1}{2}\left(J_{s \tau}^{02}+J_{s \tau}^{03}-J_{s \tau}^{23}\right), \\
& S_{e \mu}^{a}=\frac{1}{2}\left(-J_{e \mu}^{12}-J_{e \mu}^{13}-J_{e \mu}^{23}\right), \\
& S_{s e}^{b}=\frac{1}{2}\left(-J_{s e}^{02}-J_{s e}^{13}\right), \\
& S_{s \tau}^{b}=\frac{1}{2}\left(-J_{s \tau}^{02}+J_{s \tau}^{03}+J_{s \tau}^{23}\right), \\
& S_{e \mu}^{b}=\frac{1}{2}\left(J_{e \mu}^{12}-J_{e \mu}^{13}-J_{e \mu}^{23}\right), \\
& S_{s e}^{c}=\frac{1}{2}\left(J_{s e}^{02}-J_{s e}^{13}\right), \\
& S_{s \tau}^{c}=\frac{1}{2}\left(J_{s \tau}^{02}+J_{s \tau}^{03}+J_{s \tau}^{23}\right), \\
& S_{e \mu}^{c}=\frac{1}{2}\left(-J_{e \mu}^{12}-J_{e \mu}^{13}+J_{e \mu}^{23}\right) .
\end{aligned}
$$


In terms of six flavor mixing angles and three independent phase combinations of $V$, we have expressed the nine independent $J_{\alpha \beta}^{i j}$ appearing on the right-hand side of Eq. (16) in Ref. [9]. Then one may directly obtain the explicit expressions of the nine-independent $S_{\alpha \beta}^{q}$ in terms of the same mixing angles and $C P$-violating phases. For illustration, we instructively take $s_{02}, s_{03}, s_{12}, s_{13} \sim \epsilon \ll 1$ [16]. In this approximate but simpler case, we arrive at

$$
\begin{aligned}
& S_{s e}^{a} \approx \frac{1}{2}\left(-c_{01} s_{01} s_{02} s_{12} \sin \phi_{z}-c_{01} s_{01} s_{03} s_{13} \sin \phi_{y}\right), \\
& S_{s \tau}^{a} \approx \frac{1}{2}\left[c_{23} s_{02} s_{03} s_{23} \sin \phi_{x}+c_{01} c_{23}^{2} s_{01} s_{03} s_{13} \sin \phi_{y}+c_{01} s_{01} s_{02} s_{12} s_{23}^{2} \sin \phi_{z}\right. \\
& \left.+c_{01} c_{23} s_{01} s_{02} s_{13} s_{23} \sin \left(\phi_{x}-\phi_{y}\right)-c_{01} c_{23} s_{01} s_{03} s_{12} s_{23} \sin \left(\phi_{x}+\phi_{z}\right)\right] \text {, } \\
& S_{e \mu}^{a} \approx-\frac{1}{2}\left[c_{01} c_{23}^{2} s_{01} s_{02} s_{12} \sin \phi_{z}+c_{01} s_{01} s_{03} s_{13} s_{23}^{2} \sin \phi_{y}+c_{01} c_{23} s_{01} s_{03} s_{12} s_{23} \sin \left(\phi_{x}+\phi_{z}\right)\right. \\
& \left.-c_{01} c_{23} s_{01} s_{02} s_{13} s_{23} \sin \left(\phi_{x}-\phi_{y}\right)+c_{23} s_{12} s_{13} s_{23} \sin \left(\phi_{x}-\phi_{y}+\phi_{z}\right)\right] \text {, } \\
& S_{s e}^{b} \approx \frac{1}{2}\left(c_{01} s_{01} s_{02} s_{12} \sin \phi_{z}-c_{01} s_{01} s_{03} s_{13} \sin \phi_{y}\right) \text {, } \\
& S_{s \tau}^{b} \approx \frac{1}{2}\left[\left(c_{01}^{2}-s_{01}^{2}\right) c_{23} s_{02} s_{03} s_{23} \sin \phi_{x}+c_{01} c_{23}^{2} s_{01} s_{03} s_{13} \sin \phi_{y}-c_{01} s_{01} s_{02} s_{12} s_{23}^{2} \sin \phi_{z}\right. \\
& \left.-c_{01} c_{23} s_{01} s_{02} s_{13} s_{23} \sin \left(\phi_{x}-\phi_{y}\right)-c_{01} c_{23} s_{01} s_{03} s_{12} s_{23} \sin \left(\phi_{x}+\phi_{z}\right)\right] \text {, } \\
& S_{e \mu}^{b} \approx-\frac{1}{2}\left[-c_{01} c_{23}^{2} s_{01} s_{02} s_{12} \sin \phi_{z}+c_{01} s_{01} s_{03} s_{13} s_{23}^{2} \sin \phi_{y}-c_{01} c_{23} s_{01} s_{03} s_{12} s_{23} \sin \left(\phi_{x}+\phi_{z}\right)\right. \\
& \left.-c_{01} c_{23} s_{01} s_{02} s_{13} s_{23} \sin \left(\phi_{x}-\phi_{y}\right)+\left(s_{01}^{2}-c_{01}^{2}\right) c_{23} s_{12} s_{13} s_{23} \sin \left(\phi_{x}-\phi_{y}+\phi_{z}\right)\right] \text {, } \\
& S_{s e}^{c} \approx \frac{1}{2}\left(-c_{01} s_{01} s_{02} s_{12} \sin \phi_{z}-c_{01} s_{01} s_{03} s_{13} \sin \phi_{y}\right), \\
& S_{s \tau}^{c} \approx \frac{1}{2}\left[-c_{23} s_{02} s_{03} s_{23} \sin \phi_{x}+c_{01} c_{23}^{2} s_{01} s_{03} s_{13} \sin \phi_{y}+c_{01} s_{01} s_{02} s_{12} s_{23}^{2} \sin \phi_{z}\right. \\
& \left.+c_{01} c_{23} s_{01} s_{02} s_{13} s_{23} \sin \left(\phi_{x}-\phi_{y}\right)-c_{01} c_{23} s_{01} s_{03} s_{12} s_{23} \sin \left(\phi_{x}+\phi_{z}\right)\right] \text {, } \\
& S_{e \mu}^{c} \approx-\frac{1}{2}\left[c_{01} c_{23}^{2} s_{01} s_{02} s_{12} \sin \phi_{z}+c_{01} s_{01} s_{03} s_{13} s_{23}^{2} \sin \phi_{y}+c_{01} c_{23} s_{01} s_{03} s_{12} s_{23} \sin \left(\phi_{x}+\phi_{z}\right)\right. \\
& \left.-c_{01} c_{23} s_{01} s_{02} s_{13} s_{23} \sin \left(\phi_{x}-\phi_{y}\right)-c_{23} s_{12} s_{13} s_{23} \sin \left(\phi_{x}-\phi_{y}+\phi_{z}\right)\right] \text {, }
\end{aligned}
$$

where $\phi_{x} \equiv \delta_{03}-\delta_{02}-\delta_{23}, \phi_{y} \equiv \delta_{03}-\delta_{01}-\delta_{13}$ and $\phi_{z} \equiv \delta_{02}-\delta_{01}-\delta_{12}$. In obtaining these results, the corrections of $\mathcal{O}\left(\epsilon^{3}\right)$ or smaller have been neglected. Note that all $S_{\alpha \beta}^{q}$ given in Eq. (17) are suppressed by the factors of $\mathcal{O}\left(\epsilon^{2}\right)$. Note also that $S_{s e}^{a} \approx S_{s e}^{c}$ holds, as a consequence of $J_{s e}^{23} \approx 0$ in the approximation made above.

As some direct relations between the rephasing invariants of $C P$ violation $J_{\alpha \beta}^{i j}$ and the probability asymmetries of neutrino oscillations $\Delta P_{\alpha \beta}$ have been established in Ref. [9], one can straightforwardly obtain the relations between $\Delta_{\alpha \beta}$ and $S_{\alpha \beta}^{q}$ or $S_{i j}^{q}$ with the help of Eqs. (9) and (10). For simplicity, we do not go into detail at this point. We shall not discuss possible terrestrial matter effects on the unitarity quadrangles in realistic longbaseline neutrino oscillation experiments either, because the relevant discussions given in Refs. [9,20] are completely applicable here. Furthermore, we point out that the present experimental constraints on the $4 \times 4$ neutrino mixing matrix $V$ remain too poor to reveal its structural features (such as possible symmetries or asymmetries of its off-diagonal matrix elements [21]). 
In summary, we have presented a concise classification for unitarity quadrangles of the $4 \times 4$ neutrino mixing matrix. It is found that there are totally thirty-six distinct topologies among twelve different unitarity quadrangles. Useful relations between the areas of those unitarity quadrangles and the rephasing invariants of $C P$ violation have been derived. For illustration, we have also expressed nine independent areas of the unitarity quadrangles approximately in terms of six flavor mixing angles and three $C P$-violating phases in the standard parametrization.

Finally, we remark that our analytical results are model-independent and would be very useful for a systematic study of $C P$ and $T$ violation in a variety of long-baseline neutrino oscillation experiments, if the forthcoming MiniBooNE experiment could confirm the LSND anomaly and support the scheme of four neutrino mixing.

This work was supported in part by the National Natural Science Foundation of China. 


\section{REFERENCES}

[1] Super-Kamiokande Collaboration, Y. Fukuda et al., Phys. Rev. Lett. 81, 1562 (1998); 81, 4279 (1998); S. Fukuda et al., Phys. Lett. B 539, 179 (2002); M. Shiozawa, talk given at Neutrino 2002, May 2002, Munich.

[2] SNO Collaboration, Q.R. Ahmad et al., Phys. Rev. Lett. 87, 071301 (2001); 89, 011301 (2002); 89, 011302 (2002).

[3] LSND Collaboration, C. Athanassopoulos et al., Phys. Rev. Lett. 81, 1774 (1998); Phys. Rev. C 58, 2489 (1998).

[4] An exhaustive list of the references for four-neutrino mixing can be found in the web page of C. Giunti, http://www.to.infn.it/ giunti/neutrino/.

[5] H. Murayama and T. Yanagida, hep-ph/0010178.

[6] K.S. Babu and S. Pakvasa, hep-ph/0204236.

[7] M. Maltoni, T. Schwetz, M.A. Tórtola, and J.W.F. Valle, hep-ph/0207157.

[8] R. Tayloe (MiniBooNE Collaboration), talk given at Neutrino 2002, May 2002, Munich.

[9] W.L. Guo and Z.Z.Xing, Phys. Rev. D 65, 073020 (2002).

[10] Particle Data Group, K. Hagiwara et al., Phys. Rev. D 66, 010001 (2002).

[11] H. Fritzsch and Z.Z. Xing, Prog. Part. Nucl. Phys. 45, 1 (2000); hep-ph/9912358.

[12] J.A. Aguilar-Saavedra and G.C. Branco, Phys. Rev. D 62, 096009 (2000); hepph/0007025.

[13] J. Sato, Nucl. Instrum. Meth. A 472, 434 (2000); hep-ph/0008056.

[14] Y. Farzan and A.Yu. Smirnov, Phys. Rev. D 65, 113001 (2002); hep-ph/0201105.

[15] J. Schechter and J.W.F. Valle, Phys. Rev. D 22, 2227 (1980).

[16] V. Barger, Y.B. Dai, K. Whisnant, and B.L. Young, Phys. Rev. D 59, 113010 (1999).

[17] C. Jarlskog, Phys. Rev. Lett. 55, 1039 (1985).

[18] H. Fritzsch and Z.Z. Xing, Phys. Rev. D 57, 594 (1998); Nucl. Phys. B 556, 49 (1999).

[19] F.J. Botella and L.L. Chau, Phys. Lett. B 168, 97 (1986).

[20] Z.Z. Xing, Phys. Rev. D 64, 033005 (2001); Fortsch. Phys. 50, 569 (2002).

[21] Discussions about a near off-diagonal symmetry of the $3 \times 3$ lepton flavor mixing matrix can be found in: Z.Z. Xing, Phys. Rev. D 65, 113010 (2002). 


\section{FIGURES}

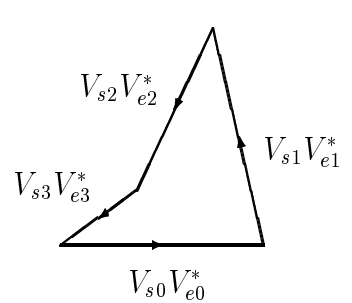

(a)

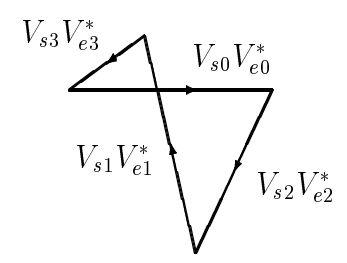

(b)

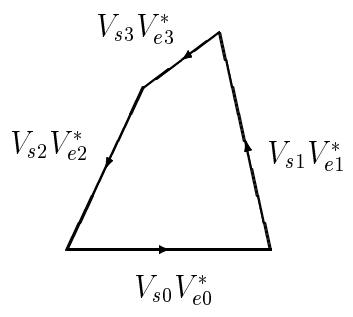

(c)

FIG. 1. Three distinct topologies of unitarity quadrangle $\mathrm{Q}_{s e}$. 